EPJ manuscript No.

(will be inserted by the editor)

\title{
Proposed search for an electric-dipole moment using laser-cooled ${ }^{171} \mathbf{Y b}$ atoms
}

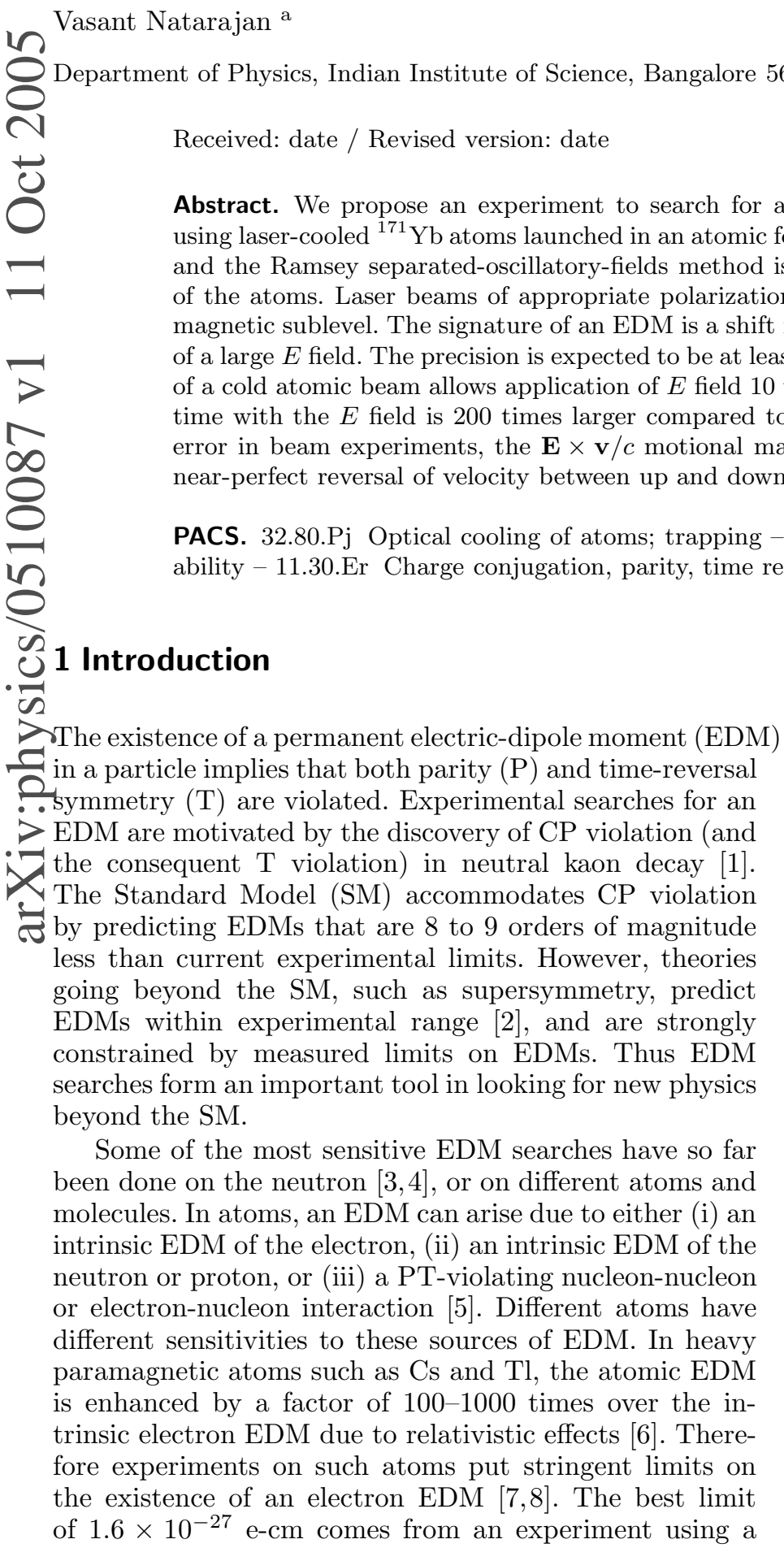

a Electronic mail: vasant@physics.iisc.ernet.in thermal beam of ${ }^{205} \mathrm{Tl}$ atoms 9 . On the other hand, diamagnetic atoms (such as $\mathrm{Hg}$ and $\mathrm{Yb}$ ) are more sensitive to the nuclear Schiff moment and possible PT-odd interactions. Experiments have been done on ${ }^{129} \mathrm{Xe}[10$ and ${ }^{199} \mathrm{Hg}\left[11\right.$ in vapor cells, with the best limit of $2.1 \times 10^{-28}$ e-cm from the ${ }^{199} \mathrm{Hg}$ experiment. Sensitive EDM searches have also been done using diatomic molecules containing heavy atoms, notably TlF [12] and YbF [13. Recently, $\mathrm{PbO}$ has been proposed as a good candidate for probing the electron EDM [14.

In this paper, we propose an experiment to search for an atomic EDM using laser-cooled ${ }^{171} \mathrm{Yb}$ launched in an atomic fountain. The experiment is performed in the presence of a uniform magnetic field that sets the quantization axis. As the atoms fall under gravity, they are first spin polarized, and then interact with an oscillating magnetic field twice, once on the way up and once on the way down. The interactions are used to perform a Ramsey separatedoscillatory-fields measurement [15] of the Zeeman precession frequency. In between the two interactions, the atoms pass through a region of large electric field, applied in a direction parallel to the magnetic field. The signature of an EDM is a shift in the Ramsey resonance correlated with the reversal of the electric field.

There are three primary advantages to our scheme. The first is that laser cooling provides a slow, dense sample of atoms that is almost purely mono-energetic. Thus the interaction time with the electric field is very long (compared to a thermal beam) and almost exactly the same for all atoms. The second advantage is that we use 
Vasant Natarajan: Proposed search for an electric-dipole moment using laser-cooled ${ }^{171} \mathrm{Yb}$ atoms

the power of the Ramsey technique to measure the Zeeman precession frequency. This ensures high precision in the frequency measurement. The third advantage is that the leading source of systematic error, namely the effect of the motional magnetic field $\left(B_{\text {mot }}=\mathbf{E} \times \mathbf{v} / c\right)$, is greatly reduced. This is because the velocity of the atoms reverses between the up and down trajectories, and the net effect is zero.

We have chosen $\mathrm{Yb}$ because it has been successfully laser cooled in our laboratory [16] and elsewhere [17.18]. It is a heavy diamagnetic atom and effects leading to EDM are comparable to that in $\mathrm{Hg}$. Furthermore, atomic calculations in $\mathrm{Yb}$, which are necessary for relating the measured EDM to fundamental PT-violating interactions, are well developed 19. Precise atomic calculations in $\mathrm{Yb}$ [20] have also been motivated by the fact that $\mathrm{Yb}$ is a promising candidate for measuring atomic parity-nonconservation effects 21,22. There are two isotopes of $\mathrm{Yb}$ that are suitable for an EDM measurement: ${ }^{171} \mathrm{Yb}(I=1 / 2)$ and ${ }^{173} \mathrm{Yb}(I=5 / 2)$. Both isotopes have roughly the same natural abundance (14.3\% and $16.1 \%$, respectively), but we have chosen ${ }^{171} \mathrm{Yb}$ because its simple magnetic-sublevel structure allows for an extremely state-selective detection scheme, as discussed later. However, it might be interesting to measure EDM in both isotopes to address nuclearinteraction related uncertainties when comparing experimental results with atomic calculations.

The use of laser-cooled atoms to measure EDM has been proposed earlier. In the early days of laser cooling, possible measurement of the electron EDM using cold Cs atoms in an atomic fountain was considered [23]. However, it was shown that Cs has potential problems with cold collisions that cause spin relaxation and lead to frequency shifts 24 . Closed-shell fermionic atoms with a ${ }^{1} S_{0}$ ground state, such as ${ }^{171} \mathrm{Yb}$, do not have these problems. Indeed, once these atoms are spin polarized, the $s$-wave scattering cross section is zero, while higher scattering cross sections go to zero at sufficiently low temperatures. Thus the spin-coherence time is of order $1000 \mathrm{~s}$ or more because effects such as three-body recombination rates and collisional spin relaxation are greatly reduced. There is a recent proposal to measure EDM using laser-cooled Cs atoms trapped in optical lattices 25. In this case, there are potential systematic effects due to AC Stark shifts in the Zeeman sublevels caused by the trapping fields. Similar Zeeman frequency shifts are present for proposed EDM experiments using cold atoms in far-detuned dipole traps. The size of these shifts have been calculated both for paramagnetic atoms (Cs) and diamagnetic atoms $(\mathrm{Hg})$ [26, showing that the experimental configuration needs to be carefully designed to control these effects. The major advantage of a fountain experiment is that there is no perturbation from trapping fields during the EDM measurement. However, it should be noted that the interaction time in a fountain experiment is limited to less than a second due to gravity, whereas the background-collision limited interaction time in an optical lattice can be 10-100 times longer.

\section{Experimental details}

Atomic EDMs are measured using spin-polarized atoms in the presence of parallel (or anti-parallel) electric (E) and magnetic (B) fields. Since the total angular momentum $(\mathbf{F})$ is the only vector quantity in the body-fixed frame, both the electric-dipole moment $(\mathbf{d})$ and the magneticdipole moment $(\boldsymbol{\mu})$ are proportional to $\mathbf{F}$. Therefore, the interaction Hamiltonian in the presence of the $\mathbf{E}$ and $\mathbf{B}$ fields is given by:

$$
H_{\text {int }}=-(d \mathbf{E}+\mu \mathbf{B}) \cdot \frac{\mathbf{F}}{F} .
$$

The Zeeman precession frequency changes when the direction of the $\mathbf{E}$ field is reversed from parallel to anti-parallel. For ${ }^{171} \mathrm{Yb}$, the nuclear spin $I$ is $1 / 2$, and an atom in the ${ }^{1} S_{0}$ ground state has $F=1 / 2$. Thus the change in the Zeeman precession frequency on $E$-field reversal is

$$
\Delta \omega_{0}=\frac{2 d E}{\hbar} .
$$

Measurement of $\Delta \omega_{0}$ therefore constitutes a measurement of the EDM $d$.

The above analysis shows that, in order to measure $d$ precisely, one needs to (i) measure the Zeeman precession frequency very precisely, (ii) have a large $E$ field, and (iii) keep the interaction time with the $E$ field as large as possible. Atomic EDM measurements are usually performed using thermal beams 927 or in vapor cells 8,11. With thermal beams, the main limitation is that the interaction time is only a few milliseconds even if the $E$-field region is $100 \mathrm{~cm}$ long. We will see later that the use of cold atoms increases the interaction time by a factor of 200 . In vaporcell experiments, the applied $E$ field is limited by the high pressure to about $10 \mathrm{kV} / \mathrm{cm}$. The use of an atomic beam allows the $E$ field to be at least 10 times higher.

The schematic of the proposed experiment is shown in Fig. 1. The atoms are first laser cooled and trapped in a magneto-optic trap (MOT). For Yb, there are two transitions that can be used for laser cooling: the ${ }^{1} S_{0} \leftrightarrow$ ${ }^{1} P_{1}$ transition at $399 \mathrm{~nm}$, and the ${ }^{1} S_{0} \leftrightarrow{ }^{3} P_{1}$ intercombination line at $556 \mathrm{~nm}$. Both lines are accessible with existing laser technology, the first using a frequency-doubled Ti-sapphire laser, and the second using a dye laser operating with Rhodamine 110 dye. We have earlier shown that a Yb MOT can be directly loaded from a thermal source (without the use of a Zeeman slower) using the $399 \mathrm{~nm}$ line 16. The source is not isotopically enriched and contains all the seven stable isotopes in their natural abundances. We are able to trap each isotope individually since the isotope shifts are about $100-1000 \mathrm{MHz}$ and the individual transitions are clearly resolved. The typical number of trapped atoms is more than $10^{8}$. In Ref. 17, $\mathrm{Yb}$ atoms emanating from an oven are first slowed in a Zeeman slower using the $399 \mathrm{~nm}$ line, and then captured in a MOT operating with the $556 \mathrm{~nm}$ line. The primary advantage of the $556 \mathrm{~nm}$ line is that its natural linewidth is only $180 \mathrm{kHz}$, which results in a Doppler cooling limit of $4 \mu \mathrm{K}$. Therefore, for the EDM experiment it is desirable to use a MOT with the $556 \mathrm{~nm}$ line. The MOT can 
be loaded directly from a Zeeman slower or from another MOT operating with the $399 \mathrm{~nm}$ line.

Once the atoms are loaded into the trap, the trapmagnetic field is turned off and the atoms are allowed to equilibriate in the optical molasses. They are then launched upwards using the standard technique of moving molasses: the detuning of the vertical beams is adjusted so that the atoms are cooled in a frame moving upwards at a velocity of $2.5 \mathrm{~m} / \mathrm{s}$. Since the energy spread in the vertical direction still corresponds to the cooling limit of $4 \mu \mathrm{K}$, the vertical velocity varies by less than $1 \%$. This means that the spread in the interaction time is only of order $1 \%$. By comparison, the velocity spread in the $\mathrm{Tl}$ experiment is $\pm 50 \%$, corresponding to the full thermal distribution at $970 \mathrm{~K}$ [9].

The EDM experiment is done in the presence of a static magnetic field of $1.33 \mathrm{G}$ that also sets the quantization axis. The resulting Zeeman precession frequency in the $F=1 / 2$ ground state is $\sim 1 \mathrm{kHz}$. For comparison the $\mathrm{Tl}$ experiment uses a magnetic field of $0.4 \mathrm{G}$, whereas the $\mathrm{Hg}$ experiment uses a field of $0.015 \mathrm{G}$.

The freely falling atoms are first spin polarized using a beam of right-circularly polarized $\left(\sigma^{+}\right)$light at $556 \mathrm{~nm}$. The laser is tuned to the $F=1 / 2 \rightarrow F^{\prime}=1 / 2$ transition. Since this is a closed transition, atoms are optically pumped into the $m_{F}=+1 / 2$ sublevel of the ground state. The atoms then pass through an interaction region consisting of a magnetic field rotating at the Zeeman precession frequency. The strength of the rotating field is adjusted such that the interaction appears as a $\pi / 2$ pulse, which puts the atoms in an equal superposition of $m_{F}=+1 / 2$ and $m_{F}=-1 / 2$ sublevels. Since the velocity spread is $\sim 1 \%$, all the atoms experience a $\pi / 2$ pulse. On the way down, the atoms interact again with the rotating field for a second $\pi / 2$ pulse. If the oscillator is exactly on resonance with the Zeeman precession frequency, the second $\pi / 2$ pulse completes the transfer of population to the $m_{F}=-1 / 2$ sublevel. This is a standard Ramsey separated-oscillatory-fields method for measuring the precession frequency [15].

Population in the $m_{F}=-1 / 2$ sublevel is detected using a probe laser on the $556 \mathrm{~nm}$ line. The probe laser is similar to the optical pumping laser: it is right-circularly polarized $\left(\sigma^{+}\right)$and drives the $\left\{F=1 / 2, m_{F}=-1 / 2\right\} \rightarrow$ $\left\{F^{\prime}=1 / 2, m_{F^{\prime}}=+1 / 2\right\}$ transition. As shown in Fig. 2 , this is an extremely state-selective detection scheme. Any atoms in the $m_{F}=+1 / 2$ sublevel of the ground state do not interact with the probe laser because the only transition from this sublevel driven by the $\sigma^{+}$light is to the $\left\{F^{\prime}=3 / 2, m_{F^{\prime}}=+3 / 2\right\}$ sublevel, which is almost $6 \mathrm{GHz}$ away 28 . Thus the laser is detuned by more than 30000 linewidths, and the transition probability is reduced by a factor of $10^{9}$. Note that the intensity of the probe can be much greater than the saturation intensity $\left(0.14 \mathrm{~mW} / \mathrm{cm}^{2}\right)$ since it is not important that atoms absorb only once from the laser. After the first excitation, atoms can decay back into either sublevel and the second excitation takes place only for those atoms that decay into the $m_{F}=-1 / 2$ sublevel. Indeed, to maximize the signal- to-noise ratio, one would like to have the atoms continue to interact with the probe laser until all of them are optically pumped into the $m_{F}=+1 / 2$ sublevel. A simple way to achieve this is to use the state-preparation beam also as the detection beam. The signal could be either from the absorbed photons or the emitted fluorescence.

In between the two $\pi / 2$ pulses, the atoms go through a region of large $E$ field (where the $B$ field is also present). Since the atoms are launched upwards with a velocity of $2.5 \mathrm{~m} / \mathrm{s}$, the height before they turn around due to gravity is $32 \mathrm{~cm}$. Therefore the $E$-field interaction can be about $30 \mathrm{~cm}$ long, corresponding to a total interaction time of $500 \mathrm{~ms}$. By comparison, the interaction time in the $\mathrm{Tl}$ experiment is only $2.4 \mathrm{~ms}$, even though the $E$-field plates are $100 \mathrm{~cm}$ long [9].

\section{Expected statistical precision}

To estimate the expected precision in the measurement, we first note that the atoms are like oscillators whose phase is being measured by the Ramsey technique. If the atom starts with an initial phase $\phi_{0}$, then after the Ramsey interrogation time $T$, the phase is given by

$$
\phi=\phi_{0}+\omega_{0} T+\phi_{n}
$$

where $\phi_{n}$ is the additional (random) phase noise due to spin decoherence. The presence of this noise limits the statistical uncertainty in each measurement. If the decoherence is a Poissonian process characterized by a time constant $\tau$, the variance in $\phi$ increases linearly with the measurement time as $T / \tau$. Therefore, the frequency measurement on each atom has an uncertainty given by $1 / \sqrt{\tau T}$. If we make a simultaneous measurement on an ensemble of $N$ atoms, then the shot-noise-limited uncertainty in the average frequency is

$$
\delta \omega_{S N}=\frac{1}{\sqrt{N \tau T}}
$$

Even though the above equation suggests that the measurement time $T$ should be increased indefinitely to minimize the noise, it is clear from Eq. (3) that $T$ should not be much larger than $\tau$, since otherwise the phase would be completely randomized by the noise. Rather, the statistical error should be reduced by repeating the measurement several times. The final error in the EDM $d$ after repeating the measurement $R$ times is:

$$
\delta d=\frac{\hbar}{2 E \sqrt{R N \tau T}} .
$$

To estimate the coherence time $\tau$ in the fountain, we note that $\mathrm{Yb}$ is a closed-shell atom and the coherence time is expected to be very long. For example, in the analogous case of $\mathrm{Hg}$, coherence times on the order of $500 \mathrm{~s}$ are achieved in a room-temperature vapor cell by using buffer gases $\left(\mathrm{N}_{2}\right.$ and $\left.\mathrm{CO}\right)$ and having paraffin-coated cell walls to minimize spin relaxation [11. On the other hand, 
in the case of paramagnetic atoms such as Cs, the coherence time in a vapor cell is only about $15 \mathrm{~ms}$ even in the presence of $\mathrm{N}_{2}$ buffer gas [8]. For laser-cooled $\mathrm{Na}$ atoms, the coherence time measured in a far-off resonance, reddetuned dipole trap is again only $15 \mathrm{~ms} 29$. However, in the same experiment, the coherence time increased by 300 times to $4.4 \mathrm{~s}$ when a blue-detuned trap of comparable depth was used. In the blue-detuned trap, atoms are repelled by the optical potential and spend most of their time in free fall. This demonstrates the advantage of having the atoms away from any trapping potential. Going by the experiments with $\mathrm{Na}$, a conservative estimate for the coherence time of $\mathrm{Yb}$ atoms in a fountain is $1000 \mathrm{~s}$, where we have assumed only a factor of two increase from the value for similar atoms in a vapor cell.

To calculate the final precision, we assume that the applied $E$ field is $100 \mathrm{kV} / \mathrm{cm}$, which is the typical field used in atomic-beam experiments. The number of atoms $N$ in the fountain can be as high as $10^{9}$. Using $\tau=1000$ $\mathrm{s}$ and $T=0.5 \mathrm{~s}$, the shot-noise limited uncertainty in the frequency measurement is $225 \mathrm{nHz}$ (or a relative precision of $2.25 \times 10^{-10}$ in the measurement of the Zeeman precession frequency). The repetition rate of the experiment can be about $0.5 \mathrm{~Hz}$. Therefore, we can reach the precision of the $\mathrm{Hg}$ experiment $\left(2 \times 10^{-28} \mathrm{e}-\mathrm{cm}\right)$ in about 18 minutes. By contrast, the $\mathrm{Hg}$ experiment takes about 300 days to achieve this precision. In 5 days, we would achieve a precision of $1 \times 10^{-29} \mathrm{e}-\mathrm{cm}$ (frequency precision of 0.484 $\mathrm{nHz}$ ), which is 20 times better than the best previous measurement. This analysis also shows the advantage of using a cold atomic beam. If we used instead a thermal beam moving at $400 \mathrm{~m} / \mathrm{s}$, the interaction time would be 0.75 $\mathrm{ms}$. Even if we assume that the coherence time is $1000 \mathrm{~s}$, the frequency uncertainty in each measurement would be $5.8 \mu \mathrm{Hz}$. This is why the $\mathrm{Tl}$ experiment has a frequency uncertainty as large as $25 \mu \mathrm{Hz}$ even after 6 days of averaging.

\section{Systematic errors - the $\mathrm{E} \times \mathrm{v}$ effect}

The above estimate of the statistical error is meaningful only if we can keep systematic errors below this level. The leading source of systematic error in any beam experiment is due to the motional magnetic field, i.e. the applied electric field appears as a magnetic field in the rest frame of the atom. If the atom is moving with a velocity $\mathbf{v}$, the total magnetic field in the atom's frame is given by:

$$
\mathbf{B}_{\text {atom }}=\mathbf{B}+\frac{1}{c} \mathbf{E} \times \mathbf{v} .
$$

The measured Zeeman precession frequency is proportional to the magnitude of this field:

$$
B_{\text {atom }}=\left[\left(\mathbf{B}+\frac{1}{c} \mathbf{E} \times \mathbf{v}\right) \cdot\left(\mathbf{B}+\frac{1}{c} \mathbf{E} \times \mathbf{v}\right)\right]^{1 / 2} .
$$

Using the fact that $B \gg|\mathbf{E} \times \mathbf{v}| / c$, we expand the square root as

$$
B_{\text {atom }} \approx B+\frac{(\mathbf{E} \times \mathbf{v}) \cdot \mathbf{B}}{c B}+\frac{(\mathbf{E} \times \mathbf{v})^{2}}{2 c^{2} B} .
$$

The first term in the above equation gives the correct Zeeman precession frequency. The third term is unimportant because it is very small and, moreover, is even under reversal of the $E$ field. However, the second term leads to a systematic error since it is odd under $E$-field reversal and mimics the EDM signal. The shift in the precession frequency due to this term is given by:

$$
\omega_{E \times v}=\gamma \frac{(\mathbf{E} \times \mathbf{v}) \cdot \mathbf{B}}{c B},
$$

where $\gamma$ is the gyromagnetic ratio, equal to $4.7288 \times 10^{3}$ $\operatorname{rad~s}{ }^{-1} \mathrm{G}^{-1}$ for ${ }^{171} \mathrm{Yb}$.

It is clear from Eq. (9) that the systematic error cancels if the sign of $\mathbf{v}$ reverses during the measurement. In the $\mathrm{Tl}$ experiment, this is achieved by using two atomic beams, one going up and the other going down, and carefully adjusting the oven temperatures for cancellation of the shift. The use of an atomic fountain has the velocity reversal built into it. During the free fall, the $z$-component of velocity at any point along the trajectory changes sign between the upward and downward trajectories. Since the $E$ and $B$ fields are nominally along the $x$ direction, the net effect is zero.

However, to account fully for the shift, we must consider that the $E$ and $B$ fields may not be perfectly in the $x$ direction, and that there are small velocity components along the $x$ and $y$ directions that do not reverse under gravity. For this analysis, we choose our coordinate system with the $z$ axis defined by gravity and the $x$ axis defined by the nominal $E$ (and $B$ ) field direction. In such a system, the residual components of the $E$ and $B$ fields arise mainly due to alignment errors while the residual velocity components arise due to two reasons: (i) finite transverse temperature in the optical molasses and (ii) misalignment between the vertical molasses beam and the gravity axis. To estimate the size of these effects, let us expand Eq. (9):

$$
\begin{aligned}
\omega_{E \times v} \approx \frac{\gamma}{c B_{x}}\left[\left(B_{x} E_{y}-\right.\right. & \left.B_{y} E_{x}\right) v_{z}+\left(B_{z} E_{x}-B_{x} E_{z}\right) v_{y} \\
& \left.+\left(B_{y} E_{z}-B_{z} E_{y}\right) v_{x}\right]
\end{aligned}
$$

The first term in the above equation is by far the dominant term and cancels under the perfect reversal of $v_{z}$. The third term is a product of two residual fields with a small velocity component, and is completely negligible. The second term is the only one we have to consider because it includes the large $x$ components of the fields:

$$
\omega_{E \times v} \approx \frac{\gamma}{c}\left(\frac{B_{z}}{B_{x}}-\frac{E_{z}}{E_{x}}\right) E_{x} v_{y} .
$$

The transverse temperature in the molasses is $4 \mu \mathrm{K}$, which results in an rms velocity of $v_{y}=1.4 \mathrm{~cm} / \mathrm{s}$. However, the mean velocity is zero, and there are equal numbers of atoms with positive and negative velocity components. Therefore, averaged over all atoms, the net effect due to the transverse temperature is zero. On the other hand, if there is a misalignment between the vertical molasses direction and the direction of gravity, atoms would be launched in a direction inclined to gravity and there 
would be a net transverse velocity component. The vertical molasses direction can be geometrically aligned with gravity to better than a part in 1000. In addition, the direction can be optimized by maximizing the number of atoms that return to the starting point in the atomic fountain. Assuming such alignment, we find the value of $v_{y}$ is $2.5 \mathrm{~mm} / \mathrm{s}$. From Eq. (11), to get a systematic shift smaller than $0.05 \mathrm{nHz}$, corresponding to an EDM of $10^{-30}$ e-cm, the values of $B_{z} / B_{x}$ and $E_{z} / E_{x}$ should be below $2.4 \times 10^{-5}$. This is quite reasonable since cancellation of transverse fields below $5 \times 10^{-7}$ has been reported in the Tl work 27. Furthermore, we have an experimental handle to measure the size of this effect since $v_{y}$ can be varied systematically by varying the direction of launch. By studying its variation over $\pm 3^{\circ}$, one should be able to measure the size of the term and, if necessary, determine the point of minimum error.

One other effect of the transverse velocity is that the atom samples a slightly different location of the field between the up and down trajectories. Using the transverse velocity of $2.5 \mathrm{~mm} / \mathrm{s}$, the difference in location is less than $1 \mathrm{~mm}$. Over this length scale, we can expect the field to be uniform and safely neglect any field gradients. Again the effect of the transverse velocity due to the finite transverse temperature would be larger but cancels when we average over all atoms.

We have analyzed the $\mathbf{E} \times \mathbf{v}$ effect in detail because this is the dominant source of systematic error in atomicbeam experiments. However, there are other sources of error such as imperfect reversal of the $E$ field, imperfect laser-beam polarization, stray magnetic fields from charging and leakage currents on the $E$-field plates, stray magnetic field from the high-voltage switch, etc. Many of these effects can be studied by the following "experimental handles": reversals of the $B$ field, the phase of the oscillating field, and the polarization of the state-preparation and detection laser. In general, the more the number of reversals, the better the discrimination against systematic effects. Furthermore, by varying the vertical launch velocity and direction, one can probe velocity-dependent systematic effects and explore different spatial regions of the apparatus.

\section{EDM Measurement in the ${ }^{3} P_{0}$ state}

There is another interesting possibility in the $\mathrm{Yb}$ system, which is that the EDM can be measured in the ${ }^{3} P_{0}$ metastable state. The lifetime of this state is very long since the $0 \rightarrow 0$ transition to the ground state is strongly forbidden. The lifetime is expected to be much longer than the $15 \mathrm{~s}$ lifetime of the nearby ${ }^{3} P_{2}$ state. The major advantage of the ${ }^{3} P_{0}$ state is that its mixing with states of opposite parity is 2.5 times larger than the ground state. The nearest state of opposite parity is the ${ }^{3} D_{1}$ state, which is only $7200 \mathrm{~cm}^{-1}$ away. This implies that PT-violating interactions leading to an EDM will be enhanced compared to the ground state.

Experimentally, measuring EDM in the ${ }^{3} P_{0}$ state of one of the odd isotopes $\left({ }^{171} \mathrm{Yb}\right.$ or $\left.{ }^{173} \mathrm{Yb}\right)$ is slightly more complicated. The state would be populated using a twophoton process (see Fig. 3) driving successively the ${ }^{1} S_{0} \rightarrow$ ${ }^{3} P_{1}$ transition $(556 \mathrm{~nm})$ and the ${ }^{3} P_{1} \rightarrow{ }^{3} D_{1}$ transition $(1.54 \mu \mathrm{m})$, which results in spontaneous decay into the ${ }^{3} P_{0}$ state with a $70 \%$ branching ratio. Detection in a magnetic sublevel would be achieved by measuring the absorption of a probe laser driving the ${ }^{3} P_{0} \rightarrow{ }^{3} D_{1}$ transition at $1.38 \mu \mathrm{m}$. Diode lasers at $1.54 \mu \mathrm{m}$ and $1.38 \mu \mathrm{m}$ are available commercially and accessing these transitions is not a problem. Theoretical calculations of the enhanced EDM in the metastable state will tell us whether the measurement is worth pursuing.

\section{Conclusions}

In summary, we have proposed a new experiment to search for a permanent electric-dipole moment using laser-cooled ${ }^{171} \mathrm{Yb}$ atoms launched in an atomic fountain. Cold diamagnetic atoms in a fountain are nearly perfect from an experimental point of view: they are in free fall under gravity and free from any trapping potential, they are in an ultra-high-vacuum environment with very few collisions, they hardly interact with each other, and they move slowly enough that the interaction time with external fields can be very long. We plan to use the Ramsey technique to measure the Zeeman precession frequency in the presence of a uniform $B$ field, which guarantees high precision in the frequency measurement. The proposal has several advantages compared to other measurement schemes: long interaction times and reduction in transit-time broadening compared to experiments using thermal beams, and use of large electric fields compared to vapor-cell experiments. The leading source of systematic error with atomic beams, the $\mathbf{E} \times \mathbf{v} / c$ motional magnetic field, is greatly reduced due to the near-perfect reversal of velocity between up and down trajectories. Other systematic effects that scale as the velocity should also be reduced since the velocity is 200 times smaller compared to a thermal beam. We estimate that a precision of $1 \times 10^{-29} \mathrm{e}-\mathrm{cm}$ is achievable with 5 days integration time, which is more than an order of magnitude better than the current limit in ${ }^{199} \mathrm{Hg}$.

\section{Acknowledgments}

This work was supported by the Department of Science and Technology, Government of India.

\section{References}

1. J. H. Christenson, J. W. Cronin, V. L. Fitch, and R. Turlay, Phys. Rev. Lett. 13, 138 (1964).

2. S. M. Barr, Int. J. Mod. Phys. A 8, 209 (1993); W. Bernreuther and M. Suzuki, Rev. Mod. Phys. 63, 313 (1991).

3. P. D. Miller, W. B. Dress, J. K. Baird, and N. F. Ramsey, Phys. Rev. Lett. 19, 381 (1967).

4. P. G. Harris et al., Phys. Rev. Lett. 82, 904 (1999). 
5. V. M. Khatsymovsky, I. B. Khriplovich, and A. S. Yelkhovsky, Ann. Phys. (Paris) 186, 1 (1988).

6. P. G. H. Sandars, Phys. Lett. 22, 290 (1966).

7. P. G. H. Sandars and E. Lipworth, Phys. Rev. Lett. 13, 718 (1964).

8. S. A. Murthy, D. Krause, Z. L. Li, and L. R. Hunter, Phys. Rev. Lett. 63, 965 (1989).

9. B. C. Regan, E. D. Commins, C. J. Schmidt, and D. DeMille, Phys. Rev. Lett. 88, 071805 (2002).

10. T. G. Vold, F. J. Raab, B. Heckel, and E. N. Fortson, Phys. Rev. Lett. 52, 2229 (1984).

11. M. V. Romalis, W. C. Griffith, J. P. Jacobs, and E. N. Fortson, Phys. Rev. Lett. 86, 2505 (2001).

12. D. Cho, K. Sangster, and E. A. Hinds, Phys. Rev. A 44, 904 (1991).

13. J. J. Hudson, B. E. Sauer, M. R. Tarbutt, and E. A. Hinds, Phys. Rev. Lett. 89, 023003 (2002).

14. D. DeMille et al., Phys. Rev. A 61, 052507 (2000).

15. N. F. Ramsey, Molecular Beams (Oxford University Press, Oxford, 1956), Chap. V.

16. U. D. Rapol, A. Krishna, A. Wasan, and V. Natarajan, Eur. Phys. J. D 29, 409 (2004).

17. T. Kuwamoto, K. Honda, Y. Takahashi, and T. Yabuzaki, Phys. Rev. A 60, R745 (1999).

18. T. Loftus, J. R. Bochinski, R. Shivitz, and T. W. Mossberg, Phys. Rev. A 61, 051401 (2000).

19. A. Dilip et al., J. Phys. B 34, 3089 (2001).

20. B. P. Das, Phys. Rev. A 56, 1635 (1997).

21. D. DeMille, Phys. Rev. Lett. 74, 4165 (1995).

22. D. F. Kimball, Phys. Rev. A 63, 052113 (2001).

23. M. Bijlsma, B. J. Verhaar, and D. J. Heinzen, Phys. Rev. A 49, R4285 (1994).

24. K. Gibble and S. Chu, Phys. Rev. Lett. 70, 1771 (1993).

25. C. Chin et al., Phys. Rev. A 63, 033401 (2001).

26. M. V. Romalis and E. N. Fortson, Phys. Rev. A 59, 4547 (1999).

27. E. D. Commins, S. B. Ross, D. DeMille, and B. C. Regan, Phys. Rev. A 50, 2960 (1994).

28. D. L. Clark, M. E. Cage, D. A. Lewis, and G. W. Greenlees, Phys. Rev. A 20, 239 (1979).

29. N. Davidson et al., Phys. Rev. Lett. 74, 1311 (1995).

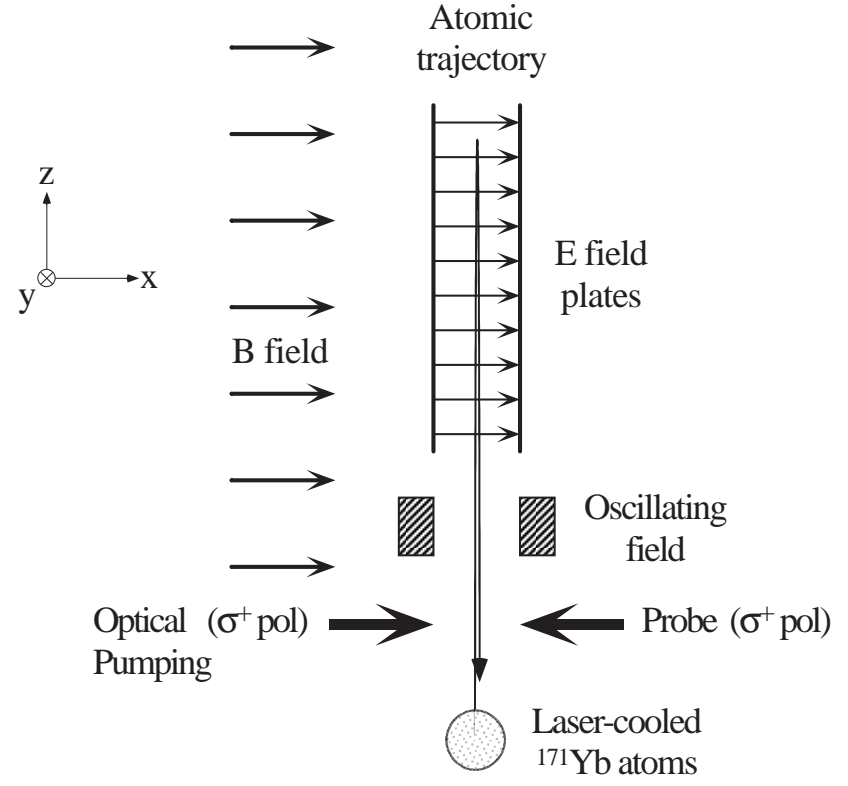

Fig. 1. Schematic of the proposed experiment. Laser-cooled ${ }^{171} \mathrm{Yb}$ atoms are launched upwards in an atomic fountain. The $z$ axis is the direction of gravity. The oscillating fields are used to measure the Zeeman precession frequency in the $B$ field. During the free fall, the atoms pass through a region of large $E$ field that shifts the precession frequency by an amount proportional to the EDM.

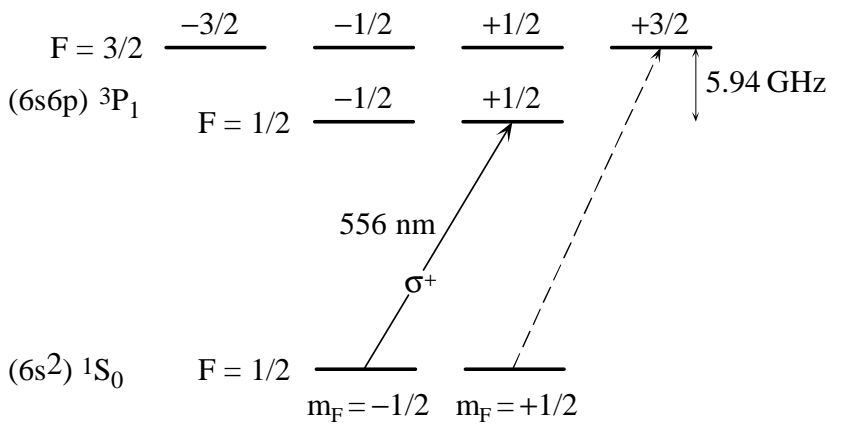

Fig. 2. State detection scheme. Atoms in the $m_{F}=-1 / 2$ sublevel are selectively detected using right-circularly polarized $\left(\sigma^{+}\right)$light at $556 \mathrm{~nm}$. The light is tuned to the $F=1 / 2 \rightarrow$ $F^{\prime}=1 / 2$ hyperfine transition. Atoms in the the $m_{F}=+1 / 2$ sublevel are not detected because the transition driven by $\sigma^{+}$ light (shown by the dashed line) is to the $F^{\prime}=3 / 2, m_{F^{\prime}}=$ $+3 / 2$ sublevel, which is $5.94 \mathrm{GHz}$ away. 


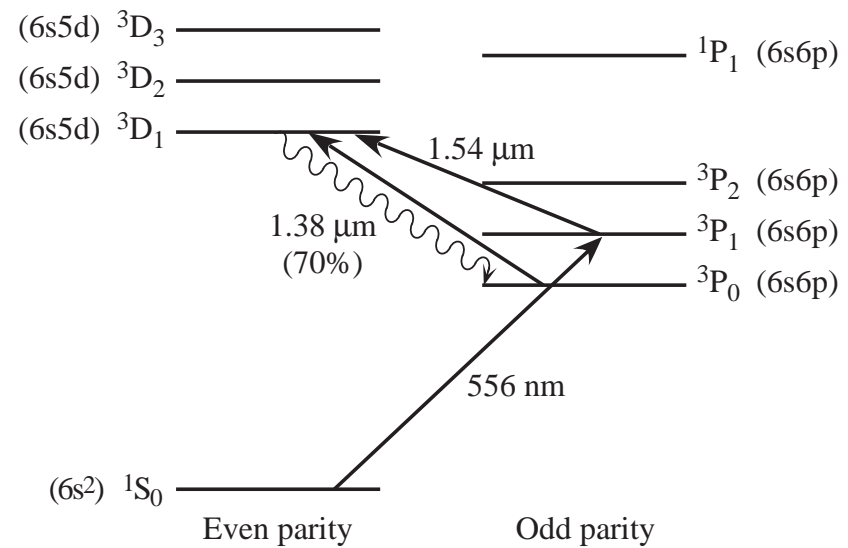

Fig. 3. Low-lying energy levels of $\mathrm{Yb}$ (not to scale) showing the two-step process for populating the metastable ${ }^{3} P_{0}$ state. The branching ratio for decay from the ${ }^{3} D_{1}$ state into the ${ }^{3} P_{0}$ state is $70 \%$. The experiment would use an odd isotope with additional hyperfine structure (not shown). State detection is by excitation back to the ${ }^{3} D_{1}$ state. 